The average number of deaths recorded per year (30.8) do not differ much from those expected (29.4), but they do occur at a younger age, in the 20-24, 25-29, and especially in the 30-34 and 35-39 age groups. This excess in the younger age groups is balanced by the lower rates in the older age groups.

A comparison between officers and servicemen demonstrates striking differences (Table I): for officers nowhere in the age groups do actual deaths exceed expected deaths. The younger age groups are almost immune up to age 39. The average expected deaths per annum are 15.2, whereas actual deaths per annum are 9.2. Therefore overall only 60.5 per cent of expected deaths occur over this five year period. For the servicemen, however, at the foot of Table I, there is a striking and substantial excess of deaths in the younger age groups up to age 39. Thereafter the observed rate equals the expected rate for the age group 40-44, and over 50 the actual deaths decline.

Expected deaths per annum are 14.1, and actual 21.6. This gives an excess of 153 per cent, a very large overall excess mortality.

Some questions inevitably arise:

a. How accurate is the diagnosis? - all deaths were coded 410 to 414 in the International Classification of Disease: more than half were confirmed by post mortem and in the remainder clinical diagnosis was thought to be accurate.

b. Is the Army, or rather are soldiers, in general less healthy (measured by mortality) than the population at large? No; when accidental deaths and deaths from IHD are excluded officers experience only 37 per cent and soldiers 59 per cent of the expected mortality for England and Wales.

\title{
Conclusion
}

These findings raise more questions than they answer. Factors requiring further investigation in the comparison between soldiers and officers are: marital status; family history of IHD; social class of parents; place of birth; (it is known that certain areas of the United Kingdom have higher death rates from IHD; some 46.9 per cent of the population in the recruiting age group live in these areas, whereas 55.2 per cent of Army recruits come from them. This may well have some effect on the soldier death rate); smoking habits (the soldier smokes significantly more than his civilian counterpart); and diet (the soldier's diet tends to be high in saturated fats and in refined sugar, not to mention chips with everything).

\section{CORONARY ATHEROSCLEROSIS IN AIRCREW}

\section{DR K E A UNDERWOOD GROUND, MRCS, LRCP, DPH, DTM\&H Royal Air Force Institute of Pathology and Tropical Medicine}

The literature on coronary atherosclerosis (CAD) in aircrew is reviewed with particular reference to Stevens' $\mathrm{s}^{1}$ and Mason' ${ }^{2}$ findings in United Kingdom aircrew. A total of 249 coronary arteries were obtained from British males killed in 172 aircraft accidents between 1968 through to the end of 1977. In addition 111 coronary arteries were examined as controls, obtained from British 
males killed in accidents unconnected with flying. The coronary arteries reviewed have been sectioned transversely, one section being taken from left main, the circumflex and the right main artery and a subjective assessment of the degree of stenosis made. The incidence of CAD among 114 military aircrew, 41 professional pilots and 84 private pilots was determined. "Significant CAD" is taken to include Grade 2 or a greater degree of stenosis and is regarded as a degree of atherosclerosis that could be 'significant' in the context of a possible cause for pilot incapacitation. The occurrence percentage of significant asymptomatic coronary atherosclerosis is given in Table II.

Table II

Incidence of Coronary artery atherosclerosis in aircrew

\begin{tabular}{llc}
\hline \multicolumn{1}{c}{ Group } & Mean age & Evidence of significant CAD \\
\hline Military aircrew (114) & 28.8 years & $16.6 \%$ \\
Commercial pilots (41) & 39.5 years & $24.3 \%$ \\
Private pilots (84) & 36.5 years & $22.6 \%$ \\
Controls (111) & 29.7 years & $18.0 \%$ \\
\hline
\end{tabular}

There is no good evidence for a statistically significant difference in the incidence of CAD in the four groups. Mason ${ }^{2}$ found that 45 ( 25 per cent) of his 180 aviators with an average age of 28.3 had Grade 2 or greater coronary atherosclerosis. There are 48 (19 per cent) Grade 2 or greater CAD in the three groups of 249 "aviators" in the present series. The observed difference in proportions between Mason's figures and the present series is not statistically signifcant: It is concluded that the incidence of CAD in the groups studied in this series has not shown any decrease in incidence over the last 15 years.

\section{The Risk Factors}

\section{A REVIEW OF NUTRITIONAL RISK FACTORS}

\section{PROFESSOR J V G A DURNIN, MA, DSc, FRCP}

The hypothesis is advanced that nutritional factors are an important component in the causation of coronary heart disease, but considerable reservation must be expressed about the quality of nutritional information provided in many of the studies relating to the aetiology of CHD. Whereas an assessment of the nutritional intake of large populations is practicable and feasible, it is not anything like so easy or so accurate for individual intake. A great deal of the difficulty in interpreting the exact role of nutrition in disease patterns stems from the problems of measuring nutritional intakes exactly.

There is a vast literature of clinical, pathological and animal experimental work to support the epidemiological findings that dietary factors are fundamental to the development of severe atherosclerosis, particularly those seen in affluent 